\title{
Measurements on quantum capacitance of individual single walled carbon nanotubes
}

\author{
Junfeng Dai, Jun Li, Hualing Zeng, and Xiaodong Cui ${ }^{\mathrm{a}}$ \\ Department of Physics, The University of Hong Kong, Hong Kong, People's Republic of China
}

(Received 23 October 2008; accepted 8 February 2009; published online 6 March 2009)

\begin{abstract}
We report measurements of the quantum capacitance of individual semiconducting and small band gap single walled carbon nanotubes (SWNTs). The observed quantum capacitance, $82 \mathrm{aF} / \mu \mathrm{m}$ for a semiconducting SWNT with chiral index $(16,8)$ and $10.3 \mathrm{aF} / \mu \mathrm{m}$ for a small band gap SWNT upon Fermi level lying at the first subband are remarkably smaller than those originating from the density of states. We attribute the discrepancy to a strong electron correlation in SWNTs and derive the Luttinger parameter $g$ of $0.25-0.3$ for the (16,8) SWNT and of 0.32 for a small band gap SWNT. (C) 2009 American Institute of Physics. [DOI: 10.1063/1.3093443]
\end{abstract}

In the classical regime, capacitance describes the object's storage capability of electric charges, and it is solely determined by electrostatic force, eventually by the geometry and the medium's dielectric constant. As the object's spatial dimensions shrink to nanometer scale where quantum effects have to be taken account, the states accommodating charges are numbered and the electric charges are regulated by the number of available states as well as electrostatic force. The Pauli exclusion principle prohibits electrons/holes from occupying the same states. Besides the modification stemming from finite density of states (DOS), electron-electron interaction may give a further correction on the quantum capacitance under some circumstance. For these nanometer scale samples and low dimensional objects such as twodimensional electron gas, ${ }^{1,2}$ nanotubes, nanowires, ${ }^{3-5}$ nanojunctions, ${ }^{2,6}$ and quantum dots, ${ }^{7,8}$ their quantum capacitance is comparable to the classical one and therefore the quantum capacitance measurement as a probe to fundamental aspects of electronic properties is possible and has been discussed theoretically and experimentally since late 1980s. Recently Ilani et al. first reported their observation of quantum capacitance of a top-gated individual carbon nanotube. ${ }^{4}$ In this letter, we present our experimental measurements on capacitance of individual microscopically long single walled carbon nanotubes (SWNTs). The measured capacitance is remarkably less than the simulated one from DOS based on tight-binding calculation, and the difference may result from the strong electron correlation.

Individual macroscopically long and diluted SWNTs were grown on a degenerately doped $\mathrm{Si} / \mathrm{SiO}_{2}$ (oxide thickness $=300 \mathrm{~nm}$ ) substrate using the chemical vapor deposition method. The catalyst was prepared by selectively dipping the diluted solution of $\mathrm{FeCl}_{3}$ on the silicon substrate and then by being reduced under $\mathrm{Ar} / \mathrm{H}_{2}$ (400 SCCM/50 SCCM) (SCCM denotes standard cubic centimeters per minute at STP) at $900{ }^{\circ} \mathrm{C}$ for $20 \mathrm{~min}$. Individual SWNTs were formed on the substrates in ethanol vapor with the same gas mixture at $900{ }^{\circ} \mathrm{C}$ for $1 \mathrm{~h}$. Electric contacts to SWNTs were made by standard optical lithography, Ti/Al metal deposition on top of as-prepared SWNTs on substrates, and finally lift off. The spacing between metal

\footnotetext{
${ }^{\text {a) }}$ Author to whom correspondence should be addressed. Electronic mail: xdcui@hku.hk.
}

electrodes varies from $10 \mu \mathrm{m}$ to hundreds of micrometers. For these semiconducting SWNTs contacting to two or more electrodes, field effect transistors (FETs) are formed with the degenerated silicon substrate as a back gate electrode. Electric characterization of these long channel (up to $1000 \mu \mathrm{m}$ ) FETs shows standard $p$-type or ambipolar behaviors at room temperature. It implies that structural discontinuity or high electric barriers due to defects are rare along SWNTs up to $1000 \mu \mathrm{m}$. The capacitance measurement was carried out by adding a small sinusoidal bias on the back gate and monitoring the displace current through the SWNT connected electrodes. To get rid of contribution from the electrodes' capacitance and the parasite capacitance of the background, we feed both displace currents from a SWNT connected electrode and from a suspended electrode of the identical geometry to a balanced amplifier, as sketch in Fig. 1. So the current arising from the capacitance of electrodes was canceled out and the metal-oxide semiconductor capacitor like behavior of silicon substrates was eliminated as well. Finally the balanced signal was fed to a lock-in amplifier, which extracts the signal amplitude and phase information, and the capacitance and resistance were analyzed. All electric measurements were carried out in vacuum condition $\left(\sim 10^{-2} \mathrm{~Pa}\right)$ at the temperature range of $77-300 \mathrm{~K}$. The geometric structure of the specific SWNTs was in situ identified by resonant micro-Raman scattering where 532 and $633 \mathrm{~nm}$ lasers were selected as excitation sources. ${ }^{9}$

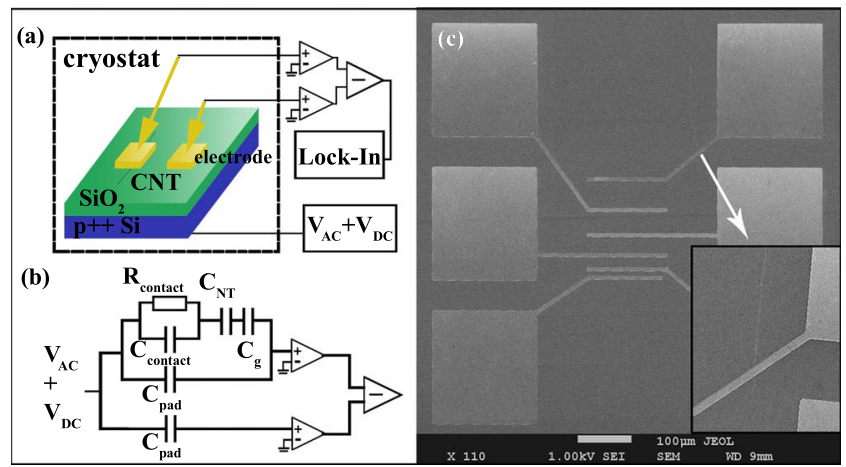

FIG. 1. (Color online) (a) Schematic of the capacitance measurement. Based on the equivalent circuit model (b), the capacitance can be derived. (c) A SEM image of devices. Inset is a zoom-in of an electrode connected with an individual SWNT (white). 

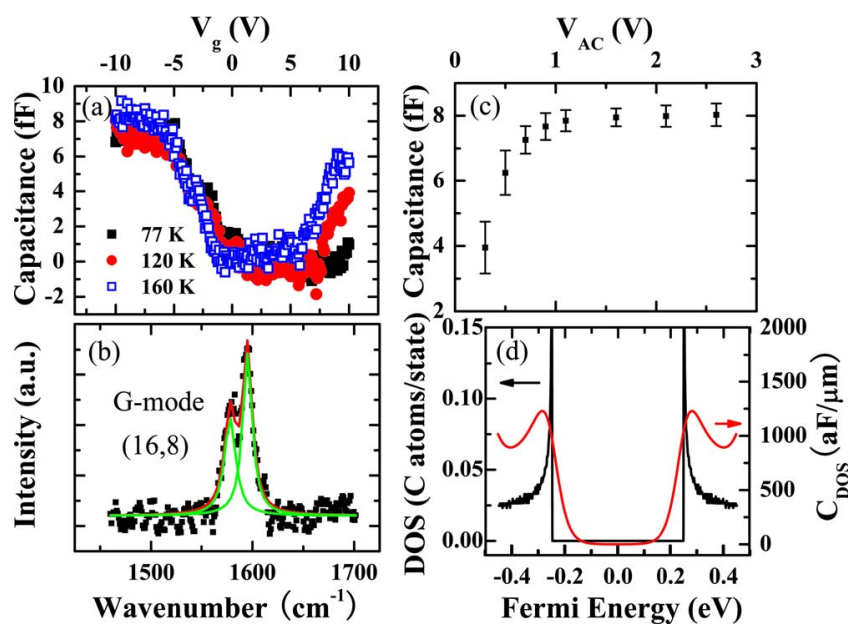

FIG. 2. (Color online) (a) The measured capacitance of a SWNT of $330 \mu \mathrm{m}$ as a function of gate voltage at various temperatures. (b) The $G$-mode Raman scattering of the SWNT with the excitation laser of $532 \mathrm{~nm}$. Radial breathing mode (RBM) cannot be obtained owing to off resonance. Two Lorentzian fittings (in green) are used to find the $\underline{G-}$ and $G+$ position. The diameter is obtained by the relation of $d=\sqrt{\omega_{G}^{+}-\omega_{G}^{-}} / C$, where $C$ $=47.7 \mathrm{~cm}^{-1}$ for semiconducting SWNTs (Ref. 9). With the aid of Kataura plot, we identified its chiral index as (16,8) (Ref. 9). (c) The measured capacitance as a function of ac bias amplitude at $77 \mathrm{~K}$. Error bars give the signal range averaged from many runs. (d) A simulated DOS of a SWNT $(16,8)$ and the corresponding capacitance arising from DOS as a function of Fermi energy, based on a zone folded tight-binding calculation.

Figure 2 shows the measured capacitance of a single SWNT $(16,8)$ as a function of $V_{g}$ at various temperatures. The curves exhibit a zero baseline at low gate bias, corresponding to the "off" state of standard SWNT-FETs. In this case, the Schottky barrier between electrodes and the SWNT prevents electrons/holes from charging and meanwhile the Fermi level lies amid the SWNT band gap and therefore no states available to accommodate charges. While $V_{g}$ increases toward either negative or positive, the Schottky barrier at electrode contacts becomes thinner and lower and the Fermi level approaches to the first subband and therefore electrons/ holes start to charge the SWNT. Consequently the capacitance exhibits remarkable increase within the gate voltage limits of $\pm 10 \mathrm{~V}$ until a plateau of $8 \mathrm{pF}$ appears at $V_{g}<$ $-6 \mathrm{~V}$, where the first subband is fully involved. Noticeably the capacitance at "on" state keeps unchanged at the temperature range of 77-160 K.

Comparing with the signal from the capacitance (the $X$ component of the lock-in out-out), the quadrature component exhibits negligible change in the whole gate voltage range at $77 \mathrm{~K}$. It implies the resistance of the whole system is negligibly small compared to $1 / \omega C(\omega=1 \mathrm{kHz})$, which is consistent with independent electric characterization, and therefore SWNTs are under equilibrium at the testing frequency even though electrons propagate diffusively along the SWNT. Since the capacitance is a measure of charging effect, whether electron transport is ballistic or diffusive does not affect the capacitance measurement as long as time scale is long enough for charging. For the presented SWNT of $330 \mu \mathrm{m}$ long measured by scanning electron microscopy (SEM), it is however questionable that there exist defects and local disorders, which act as local barriers to low energy electrons/holes. This question was examined by studying the capacitance dependence on the ac bias amplitude. Figure 2(c) shows that the on state capacitance measured at various ac

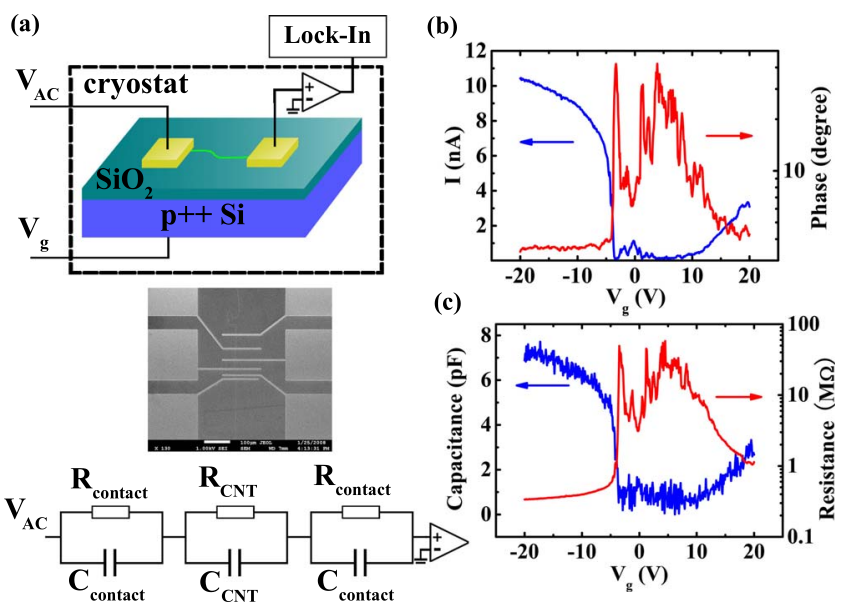

FIG. 3. (Color online) (a) Schematic of the measurement of the capacitance between electrodes and the SWNT. (b) The measured current amplitude and phase. (c) The extracted interface capacitance and contact resistance.

amplitudes keeps constant in the amplitude range of $0.8-2 \mathrm{~V}$. While the ac amplitude is lower than $0.7 \mathrm{~V}$, the capacitance drops significantly due to existence of barriers either against propagating electrons/holes or against charge injection and consequently the effective length of SWNT shrinks. Once the ac amplitude is higher than $0.8 \mathrm{~V}$, barriers vanish and the effective SWNT length expands to the whole physical length of $330 \mu \mathrm{m}$. The fact that the capacitance at on state keeps unchanged at various temperature also supports this scenario.

Another possible factor affecting our measurement on intrinsic SWNT capacitance lies in the interface capacitance between metal electrode and the SWNT, which can be treated as a serial capacitor connecting to the SWNT. To estimate this capacitance, we performed a direct measurement on several SWNT-FET structures, as sketched in Fig. 3(a). A small low frequency ac bias was fed into the drain electrode. The current through the source electrode was monitored by a lock-in amplifier. The system capacitance and the resistance could be extracted from the current amplitude and phase information according to $L R C$ circuit model. Figure 3(b) shows the conductance and phase through a SWNT-FET with a source-drain channel of $10 \mu \mathrm{m}$ as a function of $V_{g}$. Note that the phase signal at low gate voltage corresponding to off state is overwhelmed by electronic noise. Since the inductance of SWNTs is estimated at tens of $\mathrm{nH} / \mu \mathrm{m}$ (Kinetic inductance), ${ }^{10} R \gg \omega L$, its contribution to the impedance is negligible at low frequency. Based on the simplified $R C$ circuit analysis, we can obtain the interface capacitance around $10 \mathrm{pF}$ at maximum at on state, where the Schottky barrier becomes thinnest. Measurements over several SWNT-FET structures with various channel length show similar results on the interface capacitance. As the interface capacitance $(\sim \mathrm{pF})$ is three orders of magnitude greater than that the whole capacitance ( fF), as shown in Fig. 2, its contribution to the total capacitance is negligible.

To extract the quantum capacitance of single SWNT $(16,8)$, we assume that $(1) C_{\text {total }}^{-1}=C_{g}^{-1}+C_{Q}^{-1}$, where $C g$ and $\mathrm{CQ}$ are the geometric and quantum capacitance, respectively, and (2) $C_{g}=2 \pi \varepsilon L / \cosh ^{-1}(2 h / d) \approx 2 \pi \varepsilon L / \ln (4 h / d)$, where $d$ and $L$ are the SWNT diameter of $1.67 \mathrm{~nm}$ and length of $330 \mu \mathrm{m}, h=300 \mathrm{~nm}$ (further confirmed with SEM imaging) is the thickness of oxide layer and the dielectric permittivity $\varepsilon=3.9 \varepsilon_{0}$ for $\mathrm{SiO}_{2}$, and (3) the whole capacitance is sum of 


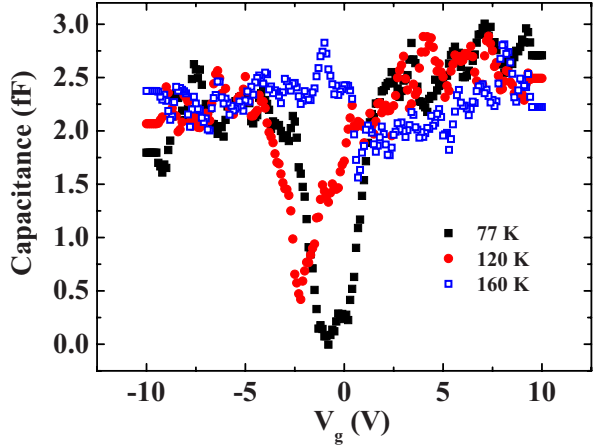

FIG. 4. (Color online) The measured capacitance of the small band gap SWNT at various temperatures.

segments in parallel. Accordingly we obtained the quantum capacitance $C_{Q}$ for the SWNT $(16,8)$ and the quantum capacitance rises from zero at low gate bias to $82 \mathrm{aF} / \mu \mathrm{m}$ at Fermi level sitting at its first subband. It is however significantly smaller than the simulated value arising from DOS calculated from the zone folded tight-binding simulation, ${ }^{11}$ as shown in Fig. 2(d), where we define

$$
C_{\mathrm{DOS}}(u)=\frac{d Q(u)}{d u}=e \int_{0}^{\infty} \frac{\partial f(V-u)}{\partial u} \times \operatorname{DOS}(V) d V .
$$

Here $f(V-u)$ denotes the Fermi-Dirac distribution and nearby carbon-carbon interaction $\gamma_{0}=2.9 \mathrm{eV}$ and overlap integral $s=0.129$ were taken in the DOS calculation. ${ }^{11}$

Figure 4(a) shows the measured capacitance as a function of $V_{g}$ of another individual SWNT. Its Raman signal could not be obtained as its optical transition does not resonate with the excitation laser, and the exact geometric index was unknown. The electric characteristic curve (conductance as a function of gate voltage, not shown) shows metallic at room temperature but semiconducting at below $160 \mathrm{~K}$. Therefore we can identify the SWNT with small band gap SWNTs which has geometric index $(n, m)$ satisfying $n-m$ $=3 \times$ integer ${ }^{12,13}$ This kind of SWNTs are expected to be metallic by tight binding calculation but are in fact semiconducting due to curvature effect that couples $P_{\pi}$ and $\sigma$ orbits to open a small gap. The capacitance measurements shown in Fig. 4(a) tell the band gap disappears above or at $160 \mathrm{~K}$. Following the similar treatment as described earlier, one can extract the quantum capacitance of $10.3 \mathrm{aF} / \mu \mathrm{m}$ for this SWNT upon the Fermi level lying in the first subband. The quantum capacitance of metallic SWNTs arising from the DOS can be estimated as follows. The energy for accommodating one electron is supposed to be equal to that of electron at Fermi level, say $\left(e^{2} / C_{\mathrm{DOS}}\right)=\hbar V_{F} K=\hbar V_{F}(2 \pi / L)$, then one gets the quantum capacitance per unit length (considering the spin degeneracy) $\left(C_{\mathrm{DOS}} / L\right)=2 e^{2} / h V_{F} \approx 96 \mathrm{aF} / \mu \mathrm{m}$, where $V_{F}$ is the Fermi velocity and is set to be $8 \times 10^{5} \mathrm{~m} / \mathrm{s}$ for metallic SWNTs.

The quantum capacitance obtained in the experiments is remarkably smaller than that from the DOS simulated with zone folded tight-binding calculation. It implies that a single particle model cannot well describe the situation here and strong electron correlation must be considered. Theoretically a one-dimensional interacting electron gas system is described by Tomonaga-Luttinger liquid instead of Fermi gas, where electron-electron interaction qualitatively instead of perturbatively modifies electric properties due to the reduced dimensionality. Its validation on carbon nanotubes was proved by independent experiments. ${ }^{14,15}$ In Luttinger liquid model, charges propagate in form of charge density wave with a velocity that is different from Fermi velocity and is determined by the strength of the interaction, saying $V_{C}$ $=V_{F} / g$, where $V_{C}$ denotes the charge velocity and $g$ is a dimensionless parameter describing the electron-electron interaction strength and therefore the compressibility of the liquid. ${ }^{16}$ As the electron correlation affect the compressibility of the liquid, the velocity and consequently the capacitance, the factor $g$ can be extracted by $g=\sqrt{C_{Q} / C_{\text {DOS. }}} \cdot{ }^{17}$ Taking this model in mind, we estimate at $77 \mathrm{~K} g$ in range of $0.25-0.3$ for SWNT $(16,8)$ and $g$ around 0.32 for the small band gap SWNT in Fig. 4 upon the Fermi level lying at the first subband. The values agree with previously reported experiments. ${ }^{4,15,18,19}$

In conclusion, we developed an experimental method to study the capacitance of individual carbon nanotubes. We obtained the quantum capacitance of $82 \mathrm{aF} / \mu \mathrm{m}$ for a semiconducting SWNT with chiral index of $(16,8)$ and of $10.3 \mathrm{aF} / \mu \mathrm{m}$ for a small band gap SWNTs upon Fermi level sitting around the first subband. The observed quantum capacitance is remarkably smaller than the capacitance originating from DOS and it implies a strong electron correlation in SWNTs. The Luttinger parameter $g$ of $0.25-0.3$ for the SWNT of $(16,8)$ and $g=0.32$ for the small band gap SWNT can be derived.

We thank Guanhua Chen, Jian Wang, and Liwei Chen for helpful discussion. The work was supported by Hong Kong GRF under Grant No. HKU 701909.

${ }^{1}$ S. Luryi, Appl. Phys. Lett. 52, 501 (1988)

${ }^{2}$ M. Buttiker, J. Phys.: Condens. Matter 5, 9361 (1993).

${ }^{3}$ J. Wang, H. Guo, J.-L. Mozos, C. C. Wan, G. Taraschi, and Q. Zheng, Phys. Rev. Lett. 80, 4277 (1998).

${ }^{4}$ S. Ilani, L. A. K. Donev, M. Kindermann, and P. L. McEuen, Nat. Phys. 2, 687 (2006).

${ }^{5}$ R. Tu, L. Zhang, Y. Nishi, and H. Dai, Nano Lett. 7, 1561 (2007)

${ }^{6}$ J. G. Hou, B. Wang, J. Yang, X. R. Wang, H. Q. Wang, Q. Zhu, and X. Xiao, Phys. Rev. Lett. 86, 5321 (2001).

${ }^{7}$ P. L. McEuen, E. B. Foxman, U. Meirav, M. A. Kastner, Y. Meir, N. S. Wingreen, and S. J. Wind, Phys. Rev. Lett. 66, 1926 (1991).

${ }^{8}$ R. C. Ashoori, H. L. Stormer, J. S. Weiner, L. N. Pfeiffer, S. J. Pearton, K. W. Baldwin, and K. W. West, Phys. Rev. Lett. 68, 3088 (1992).

${ }^{9}$ A Jorio, R. Saito, J. H. Hafner, C. M. Lieber, M. Hunter, T. D. McClure, G. Dresselhaus, and M. S. Dresselhaus, Phys. Rev. Lett. 86, 1118 (2001)

${ }^{10}$ P. J. Burke, IEEE Trans. Nanotechnol. 1, 129 (2002).

${ }^{11}$ R. Saito, G. Dresselhaus, and M. S. Dresselhaus, Phys. Rev. B 61, 2981 (2000).

${ }^{12}$ N. Hamada, S. Sawada, and A. Oshiyama, Phys. Rev. Lett. 68, 1579 (1992).

${ }^{13}$ C. Zhou, J. Kong, and H. Dai, Phys. Rev. Lett. 84, 5604 (2000).

${ }^{14}$ S. J. Tans, M. H. Devoret, R. J. A. Groeneveld, and C. Dekker, Nature (London) 394, 761 (1998).

${ }^{15}$ H. Ishii, H. Kataura, H. Shiozawa, H. Yoshioka, H. Otsubo, Y. Takayama, T. Miyahara, S. Suzuki, Y. Achiba, M. Nakatake, T. Narimura, M. Higashiguchi, K. Shimada, H. Namatame, and M. Taniguchi, Nature (London) 426, 540 (2003).

${ }^{16}$ C. L. Kane and M. P. A. Fisher, Phys. Rev. Lett. 68, 1220 (1992).

${ }^{17}$ Y. M. Blanter, F. W. J. Hekking, and M. Buttiker, Phys. Rev. Lett. 81, 1925 (1998)

${ }^{18}$ M. Bockrath, D. H. Cobden, J. Lu, A. G. Rinzler, R. E. Smalley, L. Balents, and P. L. McEuen, Nature (London) 397, 598 (1999).

${ }^{19}$ Z. Yao, H. W. C. Postma, L. Balents, and C. Dekker, Nature (London) 402, 273 (1999). 